\title{
Fractionation of respiratory and vascular responses with simple visual stimulation
}

\author{
ROBERT J. BARRY and ANGELA L. JAMES \\ School of Education, University of New South Wales, Kensington, New South Wales, Australia 2033
}

\begin{abstract}
The unitary nature of Sokolov's orienting response (OR) has been interpreted as requiring the covariation of a range of physiological indicators in response to stimulus variation. In contrast, Barry (1978) proposed a four-way fractionation of these indicators as a more realistic description of stimulus-response relationships in the OR domain. The first experiment tested aspects of this fractionation with simple visual stimulation and confirmed the existence of the three independent processes predicted for situations not requiring a motor response. Parallels between two of these processes and those of Groves and Thompson's (1970) dualprocess theory of habituation were noted, and then explored in the following two experiments. Results confirmed the fractionation obtained in the first experiment and indicated that dualprocess theory can handle various aspects of response fractionation that cannot be accommodated by Sokolov's OR theory.
\end{abstract}

Sokolov's concept of the orienting response (OR) has a number of defining characteristics that are implicit in most examples of its Western usage. In particular, the unitary nature of the OR (e.g., Sokolov, 1960, p. 191) has been interpreted as requiring that a range of physiological responsessuch as the GSR, heart rate (HR) deceleration, and changes in peripheral and cephalic pulse amplitude (PPA and CPA, respectively)-that are commonly accepted as indices of the OR (e.g., Graham, 1973) show similar stimulus-response relationships. This necessary covariation is restricted to the range of stimulus parameters at which ORs have been predicted to occur: with novel stimuli of low to moderate intensity. Within this range, OR magnitude is said to be directly related to stimulus intensity (e.g., Sokolov, 1963, p. 12) and novelty (e.g., Sokolov, 1955 , p. 134), with response enhancement at nearthreshold intensity levels. At higher intensity levels, the OR is replaced by a different reflex type, the defense reflex (DR). While much support has accumulated in the GSR literature for the validity of these stimulus-response relationships, other indices of the OR have not been generally found to exhibit such characteristics. Recent experimental reports (e.g., Maltzman, Gould, Barnett, Raskin, \& Wolff, 1979; Ray, Piroch, \& Kimmel, 1977) confirm Barry's (1977) earlier review of studies that failed to find covariation between these indices.

Barry (1979) used a factor-analytic approach to summarize a series of his studies that had investigated the unitary nature of the OR. Consistent

Address requests for reprints to Robert J. Barry, School of Education, University of New South Wales, Kensington 2033, Australia. evidence of a multifactor structure was found within the data of individual subjects, a result incompatible with the single-factor solution expected from Sokolov's unitary OR concept. This multifactor structure supported a previously hypothesized foursystem concept (Barry, 1978), which was based upon trends over group means. These four systems and their related physiological responses were: (1) stimulus register: sensitive to all stimuli (HR deceleration, CPA); (2) novelty register: sensitive to stimulus novelty (respiration, EEG); (3) intensity register: sensitive to stimulus intensity (SRR, PPA); and (4) response system: sensitive to organism's motor responses (HR acceleration). There was a considerable range of individual differences obtained in the above analysis, mainly attributed to a wide variation in within-subject correlations between the systems. Mean correlations approached zero, indicating that, on the average, the response systems were only loosely coupled, even though this did not apply at the level of the individual.

This four-fold system was tentatively offered as an alternative to Sokolov's unitary OR. Clearly, such a new hypothetical structure requires significant replication and extension of the experiments from which it was generated, in order to improve the data base. James and Barry (1980) selected one response measure from each of the first three systems listed above and investigated the effects upon these measures of stimulus magnitude and novelty. This study, while focused primarily upon the psychophysiological responding of autistic children, attempted to extend the multifactor system from the auditory modality, upon which it was based, to the visual modality. The specific predictions of relevance here were: (1) CPA (from the stimulus register) would 
fail to show either stimulus magnitude or novelty effects; (2) respiratory pause (from the novelty register) would show a novelty effect, but no stimulus magnitude effect; (3) PPA (from the intensity register) would show a stimulus magnitude effect, but no novelty effect. Separate groups of retarded children, normal schoolchildren, preschoolers, and university students generally confirmed these predictions, although the effect of stimulus magnitude obtained with PPA (while in the predicted direction) failed to reach statistical significance. While it appears likely that the relatively small difference in stimulus magnitude used in that study underlies the failure of the PPA to significantly discriminate between the different stimuli, this is a post hoc explanation, and the study provided only partial support for the multifactor structure. The present group of experiments was designed to further investigate this structure.

\section{EXPERIMENT 1}

James and Barry (1980) failed to obtain significant stimulus magnitude effects with white circles of 7.6 and $11.4 \mathrm{~cm}$ diameter, presented on a small video monitor. The first experiment increased the size difference between the large and small stimuli and presented them in an alternating sequence. The specific predictions for the nonresponse registers are as outlined above in relation to the previous experiment. They contrast with those of a unitary OR theory, which stipulates covariation of the indices, showing similar stimulus-magnitude and novelty effects.

\section{Method}

Fourteen subjects under 25 years of age volunteered to participate in the experiment to gain additional credit in an undergraduate course in educational psychology at the University of New South Wales. They were seated in a darkened room in a comfortable armchair approximately $70 \mathrm{~cm}$ in front of a Sony video monitor with a $22.5 \times 17.5 \mathrm{~cm}$ screen. Stimuli consisted of white circles, .6 and $8.5 \mathrm{~cm}$ in diameter on a black background. These were presented in an alternating sequence of 20 stimuli, with half the subjects being presented with the larger stimulus on Trial 1 . Interstimulus intervals varied randomly between 30 and $50 \mathrm{sec}$, with a mean of $40 \mathrm{sec}$. Stimulus duration was $2.0 \mathrm{sec}$.

Respiration was recorded on a Stoelting Multiscribe, with a paper speed of $2.5 \mathrm{~mm} / \mathrm{sec}$, from a Stoelting bellows pneumograph attached around the subject's chest. PPA was recorded from a Biocom piezoelectric pulse-pickup attached to the distal volar surface of the third finger of the subject's right hand (which rested on the arm of the subject chair). Another pulse-pickup was placed against the zygoma, where a pulse could be manually felt. This pulse-pickup was held in position by a pair of earphones, and its output was recorded as the CPA. Stimulus events were indicated on a fourth channel of the Multiscribe.

Subjects were told that the experiment was to investigate their physiological responses to visual stimuli and were asked to remain alert and to watch the screen for that purpose.

\section{Results}

The respiratory response was scored as the percentage increase in period of the respiratory cycle con- taining the stimulus onset compared with that of the immediately preceding cycle. This response measure, and the others employed in this study, followed previous usage in this laboratory. These measures were submitted to a two-way repeated-measures ANOVA following Poor (1973). Within trials, linear and quadratic trends were analyzed. There was a significant ${ }^{1}$ trials effect indicated by a linear trend $[F(1,12)=$ 27.82, $\mathrm{MSe}=392.15$ ]. This result is displayed in Figure 1 as a function of blocks of four trials for clarity. There was no significant stimulus-magnitude effect $[\mathrm{F}(1,12)=.20, \mathrm{MSe}=150.65]$, as indicated in Figure 1.

The PPA response was scored as the percentage decrease in pulse amplitude from the prestimulus pulse to the smallest pulse occurring between Beats 7 and 12 poststimulus. These values were analyzed in the same way as respiratory pause. Linear and quadratic trends over trials were not significant $[F(1,12)=$ $.03, \mathrm{MSe}=81.26$, and $\mathrm{F}(1,12)=3.33$, $\mathrm{MSe}=71.14$, respectively]. There was a significant magnitude effect $[F(1,12)=18.61, M S e=182.27]$, as shown in Figure 1.

CPA responses were taken as the percentage increase in pulse amplitude from the immediately prestimulus pulse to the largest pulse in the first six poststimulus beats. Analysis failed to indicate any significant effects, either over trials $[\mathrm{F}(1,12)=4.65, \mathrm{MSe}=90.52$, and $\mathrm{F}(1,12)=.36$, MSe $=109.49$, for linear and quadratic trends, respectively] or stimulus magnitude $[\mathrm{F}(1,12)=1.00, \mathrm{MSe}=170.99]$. Mean results for CPA are also displayed in Figure 1.

\section{Discussion}

These results have confirmed the predictions of response fractionation outlined in the introduction, supporting the multifactor system proposed by Barry (1979). In contrast, they cannot be accommodated by Sokolov's unitary OR theory, since the three mea-
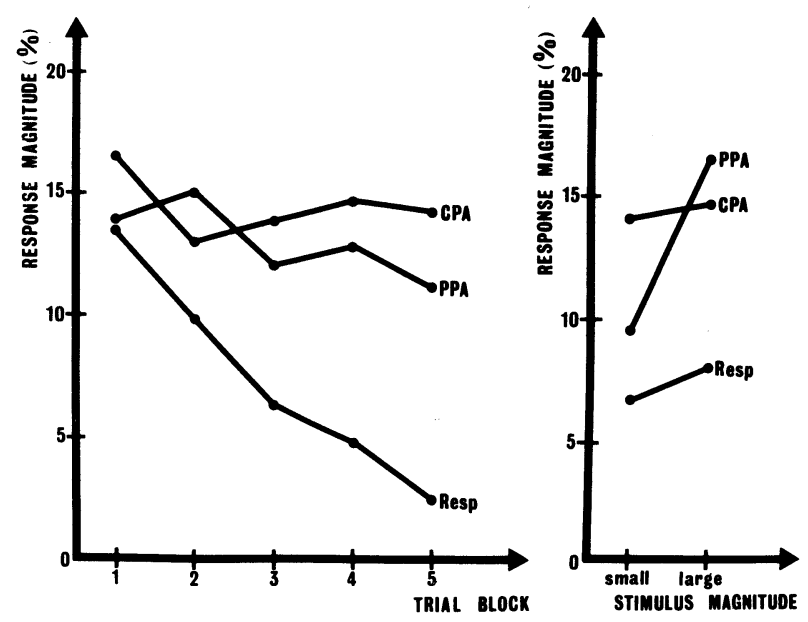

Figure 1. Response magnitudes in Experiment 1 as a function of trial block and stimulus magnitude. 


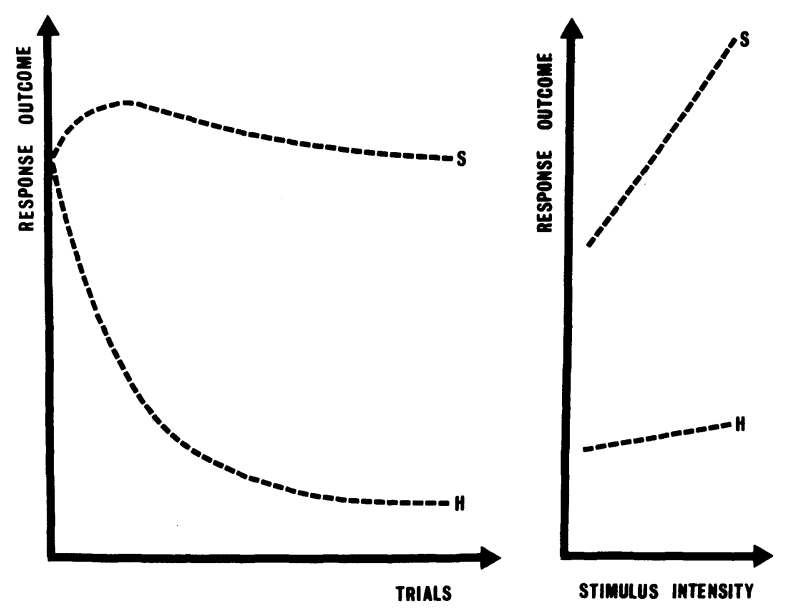

Figure 2. $S$ and $H$ as a function of stimulus repetition and intensity.

sures exhibit different stimulus-response relationships and none show both the magnitude and novelty effects predicted by that theory.

There are interesting parallels between these results and some aspects of the dual-process theory of habituation advanced by Groves and Thompson (1970). Their theory proposed that the observed habituation of a response such as the GSR was the result of an interaction between two hypothetical processes, termed habituation $(\mathrm{H})$ and sensitization $(\mathrm{S}) . \mathrm{H}$ was conceptualized as an inhibitory process strongly increasing with stimulus presentation and only weakly affected by stimulus intensity. $\mathrm{S}$ was conceptualized as a process reflecting the state of the organism, with weak enhancement produced by the first few of a series of stimuli, followed by a gradual return to baseline; this process was strongly affected by stimulus intensity. The response outcomes produced by the separate action of these processes are indicated in Figure 2. It would appear from a comparison of Figures 1 and 2 that respiratory pause shows stimulus-response relationships similar to those proposed for the outcome of process H. Also, PPA appears to show a strong stimulusmagnitude effect as proposed for $\mathrm{S}$, and there is no evidence here to contradict the predicted weak trials effect. It is thus tempting to hypothesize that respiratory pause and PPA reflect the processes of $\mathrm{H}$ and $\mathrm{S}$, respectively, and to investigate whether the predictions of dual-process theory can describe stimulus-response relationships in these indices.

\section{EXPERIMENT 2}

This experiment aimed to extend the data from Experiment 1 by presenting subjects with the same two sizes of visual stimuli in a randomized order rather than in the alternating sequence used above. Sokolov's (1963) OR theory would predict that the neuronal model required for a randomly ordered stim- ulus sequence should be more difficult to form than the neuronal model associated with a simple alternating sequence. This would result in a slower habituation of ORs elicited in this experiment as compared with the last. It is, however, illogical to apply such a prediction to measures that demonstrably do not satisfy the requirements of $O R$ indices. That is, while Sokolov's theory makes predictions about ORs elicited in these two experiments, there is no reason to apply such predictions to the indices being used.

Dual-process theory makes specific predictions for each process as a function of interstimulus interval and intensity rather than invoking such a complex hypothetical construct as the neuronal model. Generalization of $\mathrm{H}$ and $\mathrm{S}$ from one stimulus size to the other is attributed in the theory to "common elements" activated by the two stimuli. Since the magnitudes of the present stimuli are in the ratio of approximately 200 to 1 , a weak effect could be predicted since presumably few common elements are involved. Thus, the stimulus series can be considered as virtually two independent series of large and small circles intermixed. Because this experiment and the last used stimuli of the same magnitudes, it is necessary to consider only the temporal effects of randomizing rather than alternating the mix of the two stimuli.

We can expect no direct effect of presentation rate to differentiate the experiments since the same mean intertrial interval is used in both. There is, however, one possible source of difference. In an alternating mix with a mean intertrial interval of $40 \mathrm{sec}$, the effective interval between repetitions of the same stimulus will be approximately $80 \mathrm{sec}$. In contrast, with a random mix, there will be a number of occasions when this interval will extend to approximately 120 , 160 , or even $200 \mathrm{sec}$. It is reasonable to expect that the longer periods between repetitions of identical stimuli will be associated with significant spontaneous recovery (Thompson, Groves, Teyler, \& Roemer, 1973, p. 253), resulting in a reduction in $\mathrm{H}$ in this experiment, as compared with the last. Thus, if respiratory pause reflects the $\mathrm{H}$ process, the rate of decrement of this measure should be somewhat less in the second experiment, with other results being essentially replicated.

\section{Method}

Another set of 14 subjects was drawn from the subject pool of the first experiment and similarly treated. Experiment 1 was replicated except that the stimulus sequence consisted of a quasi-random mix of the two stimulus sizes (restricted so that equal numbers of large and small stimuli were presented) rather than the simple alternating mix used previously. Again, half the group received a large stimulus on Trial 1.

\section{Results}

Respiratory pause, PPA, and CPA were scored as in the previous experiment. The random mix of stimulus sizes prevented use of the same ANOVA, so similar 

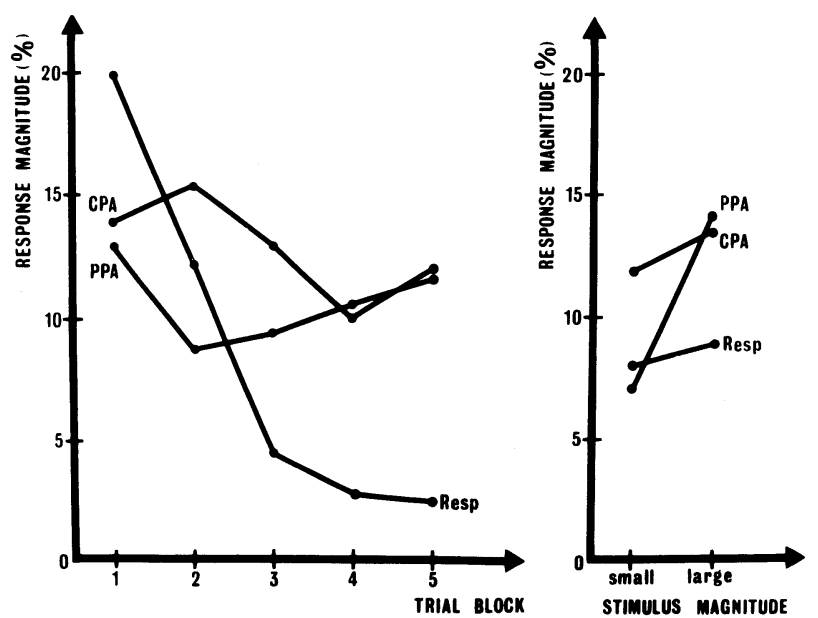

Figure 3. Response magnitudes in Experiment 2 as a function of trial block and stimulus magnitude.

one-way analyses were performed separately over trials and stimulus size. For respiratory pause, there was a significant linear trend over trials $[\mathrm{F}(1,13)=$ 20.87, $\mathrm{MSe}=198.15$ ], as illustrated in Figure 3, but no significant size effect $[\mathrm{F}(1,13)=1.38$, MSe $=$ 121.34]. With PPA, there was a significant stimulus magnitude effect $[\mathrm{F}(1,13)=75.25, \mathrm{MSe}=46.78]$, as indicated in Figure 3. CPA failed to show any significant effect of stimulus repetition or magnitude (all Fs less than unity), as illustrated in Figure 3.

The correlation coefficient between the log of the mean respiratory pause at each trial and trial number was obtained for each experiment. ${ }^{2}$ The coefficients obtained were -.92 and -.81 for Experiments 1 and 2 , respectively. These coefficients, although differing in the predicted direction, did not differ significantly.

\section{Discussion}

These results replicate those of Experiment 1, particularly in terms of the independence of the three response measures. As predicted, there was a reduced decrement rate for respiratory pause, as compared with the previous experiment, although this failed to reach statistical significance. Thus, respiratory pause and PPA responses have exhibited the stimulus-response relationships (over the parameters manipulated here) that dual-process theory (in the simplified form applied) predicted for $\mathrm{H}$ and $\mathrm{S}$, respectively. This result is sufficient to justify further exploration with these response measures.

One of the assumptions used to generate the predictions of the last experiment was that $\mathrm{H}$ would exhibit little generalization from one stimulus magnitude to the other. This was examined in the following experiment.

\section{EXPERIMENT 3}

This experiment presented visual stimuli of the same magnitudes as in the previous experiments, but in groups of one stimulus size rather than the alternating or random mixes used above. Two effects of this change are of relevance here. The first concerns the rate of repetition of the same stimulus: this is doubled from that of Experiment 1 for the same interstimulus interval. According to dual-process theory, $\mathrm{H}$ will thus develop at double the rate obtained in the first experiment. The second effect concerns the concept of generalization of $\mathbf{H}$ and $\mathrm{S}$. The change of stimulus magnitude at the end of one group of stimuli allows a direct observation of the amount of generalization obtained and a comparison with the negligible generalization assumed above.

\section{Method}

Twenty-eight subjects from the same subject pool were used in this experiment. The procedure of the previous experiments was repeated except that the stimulus sequence consisted of a group of 10 stimuli of one size followed by a group of 10 stimuli of the other size. Alternate subjects were presented with different stimulus sizes on Trial 1.

\section{Results}

Response measures were scored in the same way as in the previous experiments. The results were submitted to a three-way ANOVA following Poor (1973). Analysis was carried out over starting size, trials, and stimulus group, with repeated measures on the last two variables. Within trials, linear and quadratic trends were analyzed. With respiratory pause, the only effect to reach significance was the linear trend over trials $[\mathrm{F}(1,26)=56.97, \mathrm{MSe}=116.72]$, with stimulus group $[\mathrm{F}(1,26)=2.08, \mathrm{MSe}=192.91]$ and the interaction of stimulus group and starting size $[\mathrm{F}(1,26)=$ 2.01] failing to approach significance. (The interaction of stimulus group and starting size reflects the effect of stimulus magnitude over all trials and subjects.) These results are shown in Figure 4. No other effects or interactions approached significance. With PPA, there was a significant stimulus magnitude effect $[F(1,26)=15.11, \mathrm{MSe}=95.51]$, with no other effects or interactions approaching significance. This is shown in Figure 4. Finally, with CPA, there was a significant effect of starting size $[\mathrm{F}(1,26)=11.34$, $\mathrm{MSe}=55.16]$, with those subjects receiving the larger stimulus first exhibiting a greater response $(10.12 \%$ vs. $8.01 \%)$. Figure 4 indicates that this is not attributable to a simple magnitude effect. No other effect or interaction approached significance.

\section{Discussion}

Respiratory pause appears to have occurred in this experiment as predicted by dual-process theory. Specifically, it was predicted that little generalization from one stimulus size to the other would occur. Figure 4 confirms this, since the results for each stimulus group are very similar. It was also predicted that the decrement rate for respiration here would be approximately twice that of Experiment 1. A comparison of Figures 1 and 4 confirms this, since similar gradients of re- 

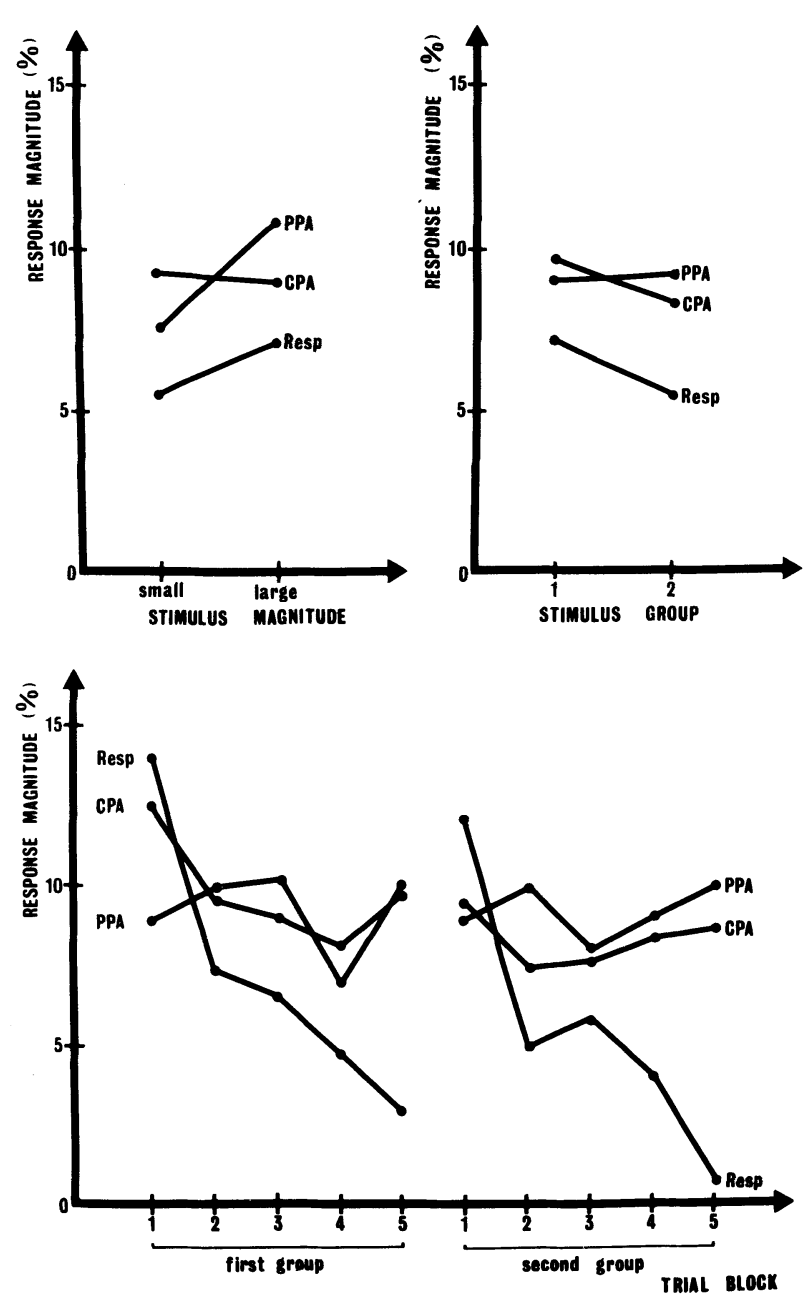

Figure 4. Response magnitudes in Experiment 3 as a function of trial block, stimulus group, and stimulus magnitude.

sponse magnitude vs. trial block have been obtained (note that, in Figure 1, blocks of four trials were used, while blocks of two trials were used in Figure 4). Finally, PPA exhibited a stimulus-magnitude effect similar to that obtained in the previous experiments. In contrast, CPA was significantly greater for those subjects who received the larger stimulus first, an effect that cannot be simply conceptualized since there was no overall stimulus-magnitude effect. This result cannot be explained and will not be discussed further. With this exception, all effects were as predicted, confirming the fractionation of the response measures previously obtained. In addition, the predictions generated from dual-process theory regarding the rate of response decrement of respiratory pause and generalization of this decrement from one type of stimulus to another were confirmed.

\section{GENERAL DISCUSSION}

This series of experiments was designed to investigate the fractionation of Sokolov's unitary OR and the alternative multifactor structure proposed by Barry (1979). One measure from each of three of Barry's registers was chosen: respiration from the novelty register, PPA from the intensity register, and CPA from the stimulus register. It was predicted that respiratory pause would reflect stimulus novelty but not stimulus magnitude, that PPA would reflect stimulus magnitude but not novelty, and that CPA would reflect the occurrence of the stimulus per se, independently of stimulus novelty and magnitude. These predictions were verified in each experiment. Such confirmatory results are important in the development of our multifactor structure since they considerably extend the data base from the simple tonal stimulation upon which it was founded.

Since Sokolov's OR theory cannot logically be applied to the description of a response that does not satisfy the stimulus-response relationships of an OR indicator, an alternative framework was sought to apply to the observed novelty effect in respiratory pause. The dual-process theory of Groves and Thompson (1970) proposed that habituation of a behavioral response such as the OR resulted from the interaction of two processes, termed habituation $(\mathrm{H})$ and sensitization (S). A number of parallels between $\mathrm{H}$ and respiratory pause and between S and PPA were discussed. The second and third experiments tested aspects of these parallels, particularly between $\mathrm{H}$ and respiratory pause. Experimental support for these relationships indicates that dual-process theory may be useful in explaining some aspects of the fractionation of responses consistently reported from this laboratory.

If it is hypothesized that $\mathrm{H}$ and $\mathrm{S}$ are processes reflected in the novelty and intensity registers of Barry's (1979) multiregister structure, Groves and Thompson (1970) have provided detailed parametric predictions that will allow specific tests of the hypothesis. At this time, there is no other theoretical structure available that can accommodate these aspects of OR fractionation. Thus, further investigations with these measures are of some importance to both dual-process theory and our multifactor structure.

\section{REFERENCES}

BARRY, R. J. Failure to find evidence of the unitary OR concept with indifferent low-intensity auditory stimuli. Physiological Psychology, 1977, 5, 89-96.

BARRY, R. J. Physiological changes in a reaction time task: Further problems with Sokolov's dimension of stimulus "significance." Physiological Psychology, 1978, 6, 438-444.

BARRY, R. J. A factor-analytic examination of the unitary OR concept. Biological Psychology, 1979, 8, 161-178.

Graham, F. K. Habituation and dishabituation of responses innervated by the autonomic nervous system. In H. V. S. Peeke \& M. J. Herz (Eds.), Habituation. New York: Academic Press, 1973.

Groves, P. M., \& Thompson, R. F. Habituation: A dual-process theory. Psychological Review, 1970, 77, 419-450.

JAMES, A. L., \& BARRY, R. J. Respiratory and vascular responses to simple visual stimuli in autistics, retardates and normals. Psychophysiology, 1980, 17, 541-547. 
Maltzman, I., Gould, J., Barnett, O. J., Raskin, D. C., \& WolfF, C. Habituation of the GSR and digital vasomotor components of the orienting reflex as a consequence of task instructions and sex differences. Physiological Psychology, 1979, 7, 213-220.

Poor, D. D. S. Analysis of variance for repeated measures designs: Two approaches. Psychological Bulletin, 1973, 80, 204-209.

Ray, R. L., Piroch, J. F., \& Kimmel, H. D. The effect of task and stimulus variability on habituation of electrodermal and vasomotor reactions. Physiological Psychology, 1977, 5, 189-196.

Sokolov, E. N. The higher nervous activity and the problem of perception. Acta Psychologica, 1955, 13, 134-135.

Sokolov, E. N. Neuronal models and the orienting reflex. In M. A. Brazier (Ed.), The central nervous system and behavior. Transactions of the 3rd conference. New York: Macy, 1960.
Sokolov, E. N. Perception and the conditioned reflex. Oxford: Pergamon Press, 1963.

Thompson, R. F., Groves, P. M., Teyler, T. J., \& Roemer, R. A. A dual-process theory of habituation: Theory and behavior. In H. V. S. Peeke \& M. J. Herz (Eds.), Habituation. New York: Academic Press, 1973.

\section{NOTES}

1. All tests of significance were at $p<.01$.

2. The log measure was used because dual-process theory predicts an exponential increase in $\mathrm{H}$ with stimulus repetition.

(Received for publication May 30, 1980; accepted October 20,1980.) 